\title{
EL RAZONAMIENTO SILOGÍSTICO COMO PATRÓN BÁSICO DEL PENSAMIENTO MORAL Y CIENTÍFICO*
}

\author{
THE SYLLOGISTIC REASONING AS A BASIC MODEL \\ OF THE MORAL AND SCIENTIFIC THINKING
}

\author{
Jorge Gregorio Posada Ramírez**
}

Posada R. Jorge G. Sophia Nº 7 - 2011. ISSN: 194-8932 Págs. 48-57.

Recepción: Mayo 25 de 2011

Aceptación: Julio 22 de 2011

\begin{abstract}
No puedo caracterizar mejor esos parecidos que con la expresión "parecidos de familia"; pues es así como se superponen y entrecruzan los diversos parecidos que se dan entre los miembros de una familia: estatura, facciones, color de los ojos, andares, temperamento, etc., etc. $-Y$ diré: los 'juegos' componen una familia.
\end{abstract}

Wittgenstein

RESUMEN

\begin{abstract}
El siguiente texto describe el razonamiento silogístico. Tiene el propósito de mostrar que su comprensión es fecunda para entender una de las estructuras básicas de la racionalidad de la ciencia y el comportamiento humano. Así, se establece que en tanto la ciencia es explicativa, y para las ciencias la explicación estándar se da a través del modelo nomológico deductivo, comprender el razonamiento silogístico es valioso para entender la explicación científica. De igual forma, en tanto las acciones humanas se explican a través del modelo causal de razones, el silogismo representa adecuadamente la estructura de las razones para la acción, que son valoradas desde la moral. Así, el intento de este texto es mostrar un punto de vista holístico que incluya en una misma perspectiva a la ciencia y la moralidad; se considera que esa perspectiva holística puede hacerse desde el razonamiento silogístico.
\end{abstract}

PALABRAS CLAVE

Ciencia, imagen holística, moralidad, razonamiento, silogismo.

* Las ideas expuestas en este trabajo forman parte de algunos de los temas de investigación del Grupo de investigación Razones y Acciones del Programa de Filosofía de la Universidad del Quindío, especialmente, los que se han venido desarrollando desde la línea de investigación en Filosofía de la Acción.

** Profesor Programa de Filosofía, Universidad del Quindío, Director Grupo de investigación: Razones y Acciones. gposada@ uniquindio.edu.co - Carrera 15 calle 12 Norte, Armenia, Quindío, Colombia. 


\begin{abstract}
The following text describes the syllogistic reasoning. The purpose is to show its comprehension as a fertile ground to understand the basic structures of science rationality and human behavior. Therefore it has been established that science character is explicative, and for sciences, standard explanations are given through the nomologic deductive model. The comprehension of syllogistic reasoning is valuable for the understanding of the scientific explanation. Equally, while human actions are explained through the model of causal reasons, syllogism adequately represents the structure of the reasons for the action valued from moralist perspective. In this way, the purpose of this paper is to show a holistic point of view that includes in the same perspective science and morality. It is considered that this holistic perspective can be built from the syllogistic reasoning.
\end{abstract}

Science, holistic image, morals, reasoning, syllogism.

\title{
KEY WORDS
}

\section{INTRODUCCIÓN}

Recientemente un grupo de académicos vienen publicando ensayos para hacerle frente a la crítica, por parte de filósofos y estudiosos, al pensamiento racional y a la posibilidad de la verdad. De lado de los críticos se encuentra Richard Rorty, quien afirma que la idea de verdad es análoga a la idea del Medioevo: "según la cual la moralidad es una cuestión de correspondencia con la voluntad de un Ser Divino" (Rorty, 2000 p. 21), por lo que la verdad sería al conocimiento, lo que el pecado es a la moral: un prejuicio innecesariamente dañino; en el mismo tenor, Paul Feyerabend sostiene que la racionalidad, y con ella la verdad, es sólo una tradición que mantiene el cemento de la sociedad occidental, pero que como tal no le es esencial a la naturaleza humana, pues: "No es solo que no sean necesarios ni el racionalismo ni la ciencia sino que en general no lo es ningún mito" (Feyerabend, 1985 p. 66), su filosofía tiende a mostrar que los principios más básicos asumidos por el pensamiento occidental: racionalidad y verdad no son más que: "una filosofía diabólica" (Feyerabend, 1985 p. 67). En el otro bando, en el de los defensores de la verdad y la racionalidad están filósofos como Harry Frankfurt. En su libro Sobre la verdad es generoso en descalificar la que para él es la moda intelectual de negar lo innegable: la racionalidad y la verdad. Frankfurt dice: "Estos desvergonzados antagonistas del sentido común -pertenecientes a un determinado y emblemático subgrupo que se define como "posmodernos" -niegan, con gran energía y convencimiento, que la verdad responda a algún tipo de realidad objetiva" (Frankfurt, 2007 p. 25). Frankfurt señala que tanto columnistas de periódicos, biógrafos, historiadores y un grueso grupo de académicos que niegan la posibilidad de la verdad y racionalidad, tendrían que obviar el hecho de que se construya un puente que se desploma al paso del primer carro, pues si solo hay puntos de vistas, y no hay verdad ni racionalidad, como objetarle al ingeniero imponiéndole la racionalidad sobre la resistencia de los materiales.

El siguiente trabajo separa del lado de los defensores de la racionalidad y la verdad. Desde la tradición de los filósofos que asumen a la razón, y con ella, su capacidad de razonamiento como el instrumento per ser de la naturaleza humana para lidiar con el mundo, se describirá el silogismo de Aristóteles como una de las formas básicas del razonamiento científico y moral, y a estas dos clases de razonamiento como sus prototípicas maneras de interpretación. Así, entender la estructura formal del silogismo y estas formas de interpretación, atienden el compromiso académico de tener una perspectiva holística de lo científico y lo moral promisoria para la transdiciplinariedad. Además, asumir el silogismo como uno de los patrones básicos de la racionalidad jalona el compromiso de profesores, estudiantes y académicos por el cultivo de la razón y del pensamiento lógico y formal; algunas veces injusta e inocentemente menoscabado por la creciente corriente que niega la posibilidad de lo racional y la verdad.

\section{EL RAZONAMIENTO}

Descartes en El discurso del método afirma que: "el poder de bien juzgar lo verdadero de lo falso, que es propiamente lo que se llama el buen sentido o la razón, es naturalmente igual en todos los hombres" (Descartes, 1983 p. 43), de lo que se pueden deducir dos cosas: (i) La razón es una capacidad 
natural, y en consecuencia, le es esencial al hombre y, (ii) todos los hombres la poseen sin distinción, siendo así universal; no obstante, explica Descartes, la diferencia entre unos y otros, entre los que pueden llamarse sabios y aquellos que no lo son, no radica en la razón propiamente, pues es igual en todos, sino en el uso o aplicación que unos y otros hacen de ella. Descartes es claro cuando afirma: "la diversidad de nuestras opiniones no proviene de que unos sean más razonables que otros, sino solamente de que conducimos nuestros pensamientos por diversas vías y no consideramos las mismas cosas". (Descartes, 1983 p. 43). Todo su libro, es justamente el intento por señalar cuáles deben ser las vías apropiadas para el mejor uso de la razón. Su técnica narrativa consiste en describir su búsqueda cognoscitiva como un ejemplo que ilustre a aquellos que tengan igual propósito que él: distinguir lo verdadero de lo falso, lo bueno de lo malo. Así, desde Descartes puede entenderse a la razón como una facultad natural al hombre, la facultad que le permite discernir y juzgar, y el razonamiento como el resultado de la operación de discernir y juzgar de la razón. Los razonamientos correctos son aquellos que siguen el adecuado uso de la razón, y conducen a lo bueno y verdadero, en tanto los incorrectos, son aquellos que se extravían del buen uso de la razón, conduciendo a lo que no es verdadero ni bueno.

Al asumir desde Descartes a la razón como una facultad y los razonamientos como sus productos, lo correcto o incorrecto no le es natural a la razón. Tanto en los razonamientos que llevan a lo verdadero como aquellos que conducen hacia lo falso se hace uso de la razón. Juzgar que: "hay que confiar en lo que dicen los oradores elocuentes, porque su lenguaje es galante y colorido", es tanto un razonamiento como aquel que señala que: "la verdad se presenta clara y simple, por lo que no se puede confiar en las personas elocuentes." Aunque parezcan contrarios, en ambos casos se puede ver el uso de la razón. En los dos se recrea un proceso oriundo de la razón, consistente en derivar de una información previa o dada otra información. Así, los razonamientos suelen ser definidos como el proceso de la razón mediante el cual se infiere o se deriva de una información dada, otra información.
Desde Descartes, en tanto la naturaleza humana hace que cada hombre intrínsecamente posea la razón, la capacidad de inferir, de producir razonamientos, es universal a la especie. Cuando en la prehistoria un humano tiraba su lanza contra una presa, esperando que de esto se siguiera la muerte de esta, ejecutaba, en esencia, el mismo proceso que una persona que en la actualidad marca su nombre de usuario y luego su contraseña, esperando poder entrar a su correo electrónico. En ambos casos de una información previa o dada se espera que se de otra. En la lógica, la rama de la filosofía que estudia la relación entre la información dada y la que se deriva, se entiende que: "El razonamiento es un genero especial de pensamiento en el cual se realizan inferencias, o sea que se deriva conclusiones a partir de premisas" (Copi, 1962 p.19). La intención de Descartes de prescribir la forma adecuada de guiar la razón, no se concentra en reglar como pasar de premisas, lo dado, a conclusiones, lo que se deriva. Como se anotó anteriormente, en el caso de los razonamientos que llevan a lo falso también se da esta relación. La propuesta de Descartes consistía en orientar hacia la verdad, lo sustancial de los razonamientos, su contenido no su forma.

De la anterior descripción de la razón que se hace desde Descartes se pueden colegir dos cosas: la primera es que existe un aspecto de la razón que es universal: su capacidad formal de producir razonamientos. La segunda, que la razón se hace singular en virtud a las distintas maneras como se guíe su capacidad de hacer razonamientos. Estos dos aspectos son de primera relevancia en la intención de este texto. Pues al afirmar que el silogismo es un patrón básico del razonamiento científico y moral, se asumirá el carácter universal de la razón: su facultad formal y universal de producir razonamientos, en este caso, razonamientos silogísticos. Pero a su vez, se tendrá en cuenta su carácter singular, el guiarse por distintos caminos, lo que le permite producir silogismos ya sean científicos o morales.

\section{EL RAZONAMIENTO SILOGÍSTICO}

Para Aristóteles el estudio de las ciencias, ya sean las que tratan de la materia, el cuerpo o la mente, debe estar precedido por una preparación acerca de la manera como los conceptos y las 
proposiciones se enlazan para hacer descripciones de la realidad. El Organon, reconocido como los tratados de lógica de Aristóteles, describe lo que es para Aristóteles la propedéutica necesaria antes de afrontar la investigación o el estudio de cualquier ciencia. Al ser las ciencias una representación teórica de la realidad, el estudio sobre las maneras cómo se conectan los conceptos y proposiciones es de notable importancia para las distintas ciencias. Ofrece, entonces, Aristóteles su texto El Organon el que es para algunos su libro más revolucionario, en tanto es el primer libro occidental de lógica, un estudio explícito de las formas del razonamiento humano. (Thomson, 2000 p. 9).

\section{En El Organon Aristóteles afirma:}

\begin{abstract}
El silogismo es una enunciación, en la que, una vez sentadas ciertas proposiciones, se concluye necesariamente en otra proposición diferente, solo por el hecho de haber sido aquellas sentadas. Cuando digo sólo por el hecho de haber sido sentadas las primeras proposiciones, quiero decir que a causa de ellas resulta probada la otra proposición. (Analíticos Primeros I, 1, 24 b 20-25)
\end{abstract}

El silogismo es entonces un tipo de razonamiento: dada cierta afirmación se sigue o se deriva otra. A la información dada se le reconoce como las premisas del razonamiento, en este caso, premisas del silogismo, a la información derivada, como la conclusión del silogismo. Por ejemplo, dadas las premisas: "Todos los abogados han estudiado leyes" y "Todos los jueces son abogados" se sigue que: "Todos los jueces han estudiado leyes".

No obstante, lo acentuado hasta ahora desde Aristóteles, permite que se predique de cualquier razonamiento que es silogístico, pues si el razonamiento es derivar de una información dada otra información, todo razonamiento sería silogístico. Hay un rasgo en especial mencionado en la cita de Aristóteles que empieza a demarcar la singularidad del razonamiento silogístico, y es la noción de necesidad. No todos los razonamientos conducen a que sus conclusiones se sigan necesariamente de sus premisas. En el silogismo la conclusión se sigue necesariamente de las premisas. En el caso de otros tipos de razonamiento, como son los razonamientos inductivos o analó- gicos, la conclusión no se sigue necesariamente de sus premisas. Al concluir que: "hoy va a llover en la tarde" sobre las premisas de que: "ayer al medio día el cielo se tornó gris y el viento se hizo más frio y llovió" y "hoy al medio día también se puso el cielo gris y el viento se hizo más frio", entonces "seguramente va a llover", en este caso, se está derivando de una información dada, la de ayer, otra información, lo que se supone se va a dar hoy, sólo que en este tipo de inferencia de las premisas no se sigue necesariamente la conclusión. Es posible que a pesar de lo que se sabe sobre la lluvia de ayer, hoy no llueva. En el ejemplo de los jueces, si es verdad que estos son abogados, y que los abogados estudian leyes, se sigue necesariamente que los jueces han estudiado leyes.

En consecuencia, en el caso de los razonamientos silogísticos, dadas ciertas premisas se sigue necesariamente su conclusión, por lo que la conclusión resulta enteramente probada por las premisas. En el caso de otros razonamientos, como los por analogía, dadas ciertas premisas la conclusión se sigue probablemente de sus premisas; la conclusión resulta parcialmente probada. Chain Perelman en su libro Tratado de la argumentación afirma con respecto a los argumentos por analogía: "Nadie ha negado la importancia de la analogía en la conducta de la inteligencia. Sin embargo, reconocida por todos como un factor esencia de invención, se la ha tratado con recelo tan pronto como se la quería convertir en un medio de prueba" (Perelman, 2000 p. 569). Lo que resalta el carácter de respaldo insuficiente de las premisas con respecto a la conclusión en los razonamientos por analogía, y contrasta con el respaldo total de las premisas a su conclusión en los argumentos silogísticos.

Los argumentos silogísticos son concluyentes en virtud al tipo de términos y proposiciones que lo conforman, y al tipo de relación que se da entre estos. Aristóteles dice:

Cuando tres términos están entre sí en tal relación, que el último esté en la totalidad del medio, y el medio esté o no en la totalidad del primero, es de necesidad que se forme el silogismo completo con los extremos. Llamo medio al término que, estando él mismo encerrado en otro, encierra él igualmente otro término, y se hace entonces medio por su misma posición. Los extremos son 
el término que está contenido en otro término y el término que contiene igualmente otro término.

(Analíticos Primeros, I, 4, 25 b 30-35).

En otras palabras, en esta primera descripción de los silogismos, para Aristóteles un silogismo es una estructura argumentativa conformada por tres términos: término medio, término mayor y término menor. El término medio es aquel que contiene al término menor, y a su vez, está contenido en el término mayor:

Sea $\mathrm{A}$ el termino menor, $\mathrm{B}$ el término medio, y $\mathrm{C}$ el término mayor, entonces, si todo $A$ es $B, y$ todo $B$ es $C$, entonces todo $A$ es $C$. La relación de estar contenido un término en otro se hace más clara cuando se muestra cada término como un conjunto que contiene o está contenido en otro conjunto.
Sea A

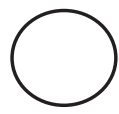

Si todo A es B,

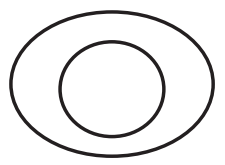

Sea C

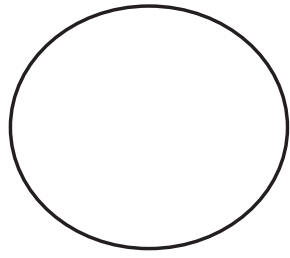

Sea B

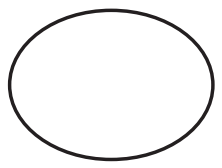

y todo $B$ es $C$,

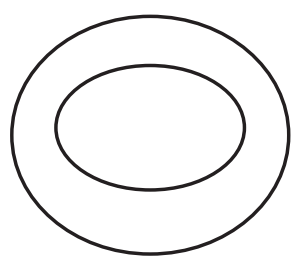

necesariamente, todo $\mathrm{A}$ es $\mathrm{C}$

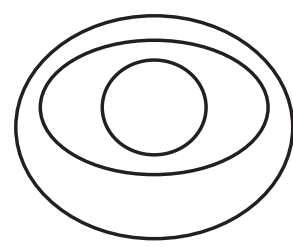

En el silogismo sobre los abogados, los jueces y las personas que han estudiado leyes, tenemos que los jueces pertenecen al conjunto de los abogados, y los abogados al conjunto de los que han estudiado derecho, por lo que los abogados son el término medio (B), los jueces el termino menor (A), y los que han estudiado el termino mayor (C).
En los razonamientos silogísticos la conclusión se sigue necesariamente de las premisas porque está contenida totalmente en ellas. La relación entre los términos que aparecen en las premisas y en la conclusión, es de tal naturaleza que afirmadas las premisas se afirma necesariamente la conclusión. Esto de nuevo, en contraste con los razonamientos por analogía o argumentos inductivos, en los que las premisas sólo afirman parcialmente lo que se dice en la conclusión. Como sostiene los lógicos, este tipo de argumentos solo son explicativos, en contraste con los razonamientos por analógica o inductivos.

Estos argumentos en los que, aun siendo correctos, la conclusión contiene más información que las premisas, son los argumentos inductivos. Así, contrariamente al carácter meramente explicativo de los deductivos, los argumentos inductivos se caracterizan por ser aumentativos. Puesto que en los argumentos inductivos válidos la conclusión contiene más información que las premisas. (Díez, 2002 p. 23)

En la cita de Aristóteles se señala que también se da la relación que el término medio no esté contenido en el término mayor, con lo que el término menor al estar contenido en el medio, queda necesariamente excluido del término mayor. En este caso también se da una conclusión necesaria, nota característica de los silogismos. "Cuando tres términos están entre sí en tal relación, que el último esté en la totalidad del medio, y el medio esté o no en la totalidad del primero, es de necesitad que se forme el silogismo completo con los extremos." (Analíticos Primeros, I, 4, 25 b 30). El subrayado es mío.

La segunda característica que singulariza a los silogismos de los demás razonamientos es el número de premisas que lo conforman. En los silogismos dos son las premisas y una la conclusión. Un razonamiento con más de dos premisas no es estrictamente lo que Aristóteles llama silogismo. Cuando Aristóteles representa formalmente el silogismo diciendo: "Si A se atribuye a todo $B, y$ B se atribuye a toda $C$, es necesario que $A$ se atribuye a toda C" (Analíticos Primeros, I, 4, 25 b 35), se ejemplifica que tres son el número de proposiciones que conforma un silogismo, dos premisas: (i) A se atribuye a todo B, y (ii) B se atribuye a todo C; y una conclusión: (iii) A se atribuye a todo C. La premisa que contiene el término mayor se le llama 
premisa mayor, y a la que tiene el término menor, premisa menor. Es de notar que el término medio no aparece en la conclusión y que se da tanto en la premisa mayor como en la menor, y que en la conclusión se da la relación del término mayor y menor.

(i) Si todo abogado ha estudiado leyes, (A se atribuye a todo $\mathrm{B}$, premisa mayor)

(ii) Y Si todo Juez es abogado ( $C$ se atribuye a todo $A$, premisa menor)

(iii) Necesariamente, todo juez ha estudiado leyes ( $\mathrm{C}$ se atribuye a todo $\mathrm{B}$, conclusión).

Esquemáticamente:

\begin{tabular}{ll}
$\begin{array}{ll}\text { Todo A es B } \\
\text { Todo C es A }\end{array}$ & Premisas \\
\hline Por lo tanto Todo C es B & \} Conclusión
\end{tabular}

Sintetizando, todo lo anterior, se mostró desde Descartes que producir razonamientos es una capacidad universal, la cual consiste en derivar de cierta información previa otra información. Desde Aristóteles que los silogismos son un tipo de razonamiento conformados por tres términos que en la relación de estar contenido o no estarlo, permiten derivar necesariamente una conclusión. Además, que están conformados por tres proposiciones, de la cuales, dos son premisas y la otra es conclusión, conclusión que se sigue necesariamente de sus premisas.

Una vez que se ha aclarado en qué consiste el razonamiento silogístico, lo que sigue en este trabajo es mostrar como el razonamiento silogístico es un elemento básico en la racionalidad científica y la racionalidad práctica, esto es, en la manera como la ciencia explica los fenómenos y en cómo las personas guían generalmente sus acciones. El propósito no es hacer un análisis detallado de la racionalidad científica y práctica, sino mostrar que en el silogismo se configura un elemento básico de la racionalidad de estos dos campos, así que su conocimiento y uso permite entender, independiente de la disciplina, la manera básica de operar de la ciencia y la conducta humana, esta última terreno de lo moral.

\section{EL SILOGISMO EN LA CIENCIA, EL MODELO NOMOLÓGICO DEDUCTIVO}

La nota característica de la ciencia es su poder explicativo. En contraste, mientras que el conocimiento del sentido común atiende a necesidades prácticas e inmediatas, y el conocimiento ideológico es normativo, la ciencia es explicativa. Al decir del filósofo de la ciencia Karl Popper, con respecto a las teorías científicas: "Las teorías son redes que lanzamos para apresar aquello que llamamos "mundo": para racionalizarlo, explicarlo y dominarlo". (Popper, 1996 p 57). En el mismo tenor, pero ya no desde el racionalismo crítico, sino desde una visión positivista* dice Ernest Nagel:

\begin{abstract}
Es el deseo de hallar explicaciones que sean al mismo tiempo sistemáticas y controladas por elementos de juicios fácticos lo que da origen a la ciencia; y es la organización y la clasificación del conocimiento sobre la base de principios explicativos lo que constituye el objetivo distintivo de las ciencias. (Nagel, 1991 p. 17).
\end{abstract}

A pesar de las enormes diferencias entre racionalistas críticos y positivistas, en ambas posiciones se concluye que la ciencia es explicativa. Siendo la ciencia la actividad humana consistente en formular teorías, la explicación científica se da a través de la organización de las proposiciones en sistemas teóricos llamados teorías científicas. En filosofía de la ciencia el modelo básico de explicación científica es llamado el modelo nomológico deductivo, sistema que muestra de qué forma se ordenan las proposiciones para que se dé la explicación.

Descrita por el filósofo de la ciencia Carl Hempel en su célebre libro La explicación científica, estudios sobre filosofía de la ciencia distingue dos componentes de la explicación científica: el explanandum $y$ el explanans. El primero alude a la proposición que describe el suceso que se quiere explicar; el segundo son las proposiciones a las que se apelan para dar cuenta del explanandum. Así, en tanto

* El Racionalismo crítico de Popper afirma que la ciencia ha progresado porque su método es el de las conjeturas y refutaciones. Por eso los científicos deben buscar siempre la refutación de sus teóricas, y con ella, la propuesta de una nueva conjetura. En el caso de los positivistas lógicos, los científicos no buscan la refutación de sus teorías sino su verificación. 
el explanandum se deduce del explanans, este último explica al primero. Para Hempel dos son los tipos de oraciones que conforman el explanans: oraciones que describen la ocurrencia de condiciones antecedentes $(E 1, \ldots E m)$ y por lo menos una oración general que describe una ley (L). El caso representativo del radiador del auto que se revienta, o el de la caída y elevación de la columna mercurial en un termómetro (Hempel, 1996 p. 248) clarifica adecuadamente cómo el explanans, en tanto permite deducir el explanandum, lo explica, citemos al respecto a Henrik Von Wright, en su libro Explicación y comprensión, muestra:

\begin{abstract}
¿Por qué reventó por la noche el radiador de mi auto? El tanque estaba lleno hasta rebosar de agua; el tapón estaba enroscado a fondo; no había puesto anticongelante; el coche se había quedado al sereno en el patio; durante la noche descendió inesperadamente la temperatura muy por debajo de cero grados. Tales fueron los antecedentes. En combinación con las leyes físicas-en particular, la ley de que el volumen de agua se dilata al helarse-, explica la suerte del radiador. (Von Wright, 1979 p. 32).
\end{abstract}

Las condiciones antecedentes (el estado en que se encontraba el radiador minutos antes de haberse reventado) y la ley (el volumen de agua se dilata al congelarse) son el explanans, fungen como premisas de un razonamiento deductivo que tiene como conclusión el acontecimiento que se quiere explicar o explanandum (el hecho de que el radiador se reventó). De esta manera, el explanandum se explica al deducirse del explanans. Esquemáticamente:

$\begin{aligned} & \mathrm{E} 1, \mathrm{E} 2, \ldots \mathrm{Ek} \text { (condiciones antecedentes) } \\ & \mathrm{L} 1, \mathrm{~L} 2, \ldots . . \mathrm{Lr} \text { (leyes) }\end{aligned}$
$\begin{aligned} & \text { (Descripción del suceso que se explica) } \\ & \text { Explanans }\end{aligned}$ Explanandum

El modelo exige que el explanandum sea una consecuencia lógica necesaria del explanans. Al estar contenido el explanandum en el explanans, dado el explanans, se sigue necesariamente el explanandum. Como puede verse, existe una similitud del razonamiento científico que explica un suceso con el silogismo, esto porque la estructura básica del modelo nomológico deductivo es un silogismo. Como se mencionó el explanans está formado por dos clases de proposiciones, las que a la luz del silogismo, son sus dos premisas. A estas se suma la tercera proposición del silogismo o conclusión, la que se refiere desde el modelo nomológico como explanandum. La relación de explicación se da en tanto las premisas del modelo nomológico contiene la conclusión, tal como se da la relación entre las proposiciones en el silogismo.

No obstante, retrotrayendo el ejemplo del radiador roto a la estructura silogística, no se puede señalar que dos enunciados forman las premisas del razonamiento explicativo. Las condiciones antecedentes van desde la descripción del tapón del radiador, el estado del recipiente, del descenso de la temperatura en la noche; y si a esto se suma, la ley de que el volumen de agua se dilata al helarse, se tiene que en el explanans no hay estrictamente dos enunciados, como lo demanda los razonamientos silogísticos. Sin embargo, es claro que los enunciados que describen las condiciones antecedentes, configuran una clase de enunciados, con propiedades lógicamente distintas, a los enunciados que describen leyes. Las condiciones antecedentes permiten la comprobación empírica, a diferencia de las leyes generales, que dentro del modelo nomológico, no describen la ocurrencia de un suceso particular, sino de una regularidad en la naturaleza. Por esto, mientras los enunciados que describen las condiciones antecedentes se evalúan como verdaderos o falsos, las leyes no permiten este tipo de evaluación, esto último cuando se presentan como parte del explanans ${ }^{\star *}$. Esto permite distinguir dos tipos de enunciados dentro del explanans, manteniendo la analogía entre las premisas del silogismo, y los enunciados que fungen dentro del modelo nomológico como explanans; analogía que es cualitativa y no numérica. Así, tanto en el modelo nomológico deductivo, como en un razonamiento silogístico hay una estructura común de derivación. De dos

\footnotetext{
** Decir que un enunciado o grupos de enunciados empíricos permiten la evaluación de verdadero o falso, implica que es posible contrastar los enunciados con hechos o acontecimientos del mundo. Los enunciados singulares, como son las condiciones antecedentes, permiten este tipo de evaluación. No ocurre lo mismo con los enunciados que describen leyes, pues dada su generalidad, estos no se refieren a algún hecho del mundo espacial y temporal.
} 
tipos de premisas, en el caso del modelo nomológico, explanans, se sigue una conclusión: el explanandum; de dos premisas, en el caso del silogismo, se sigue una conclusión. Y es a través de la relación de estar contenida o subsumida una proposición en otras, que se produce el acto de explicación o derivación.

Así, la manera estándar de explicación científica, o modelo nomológico deductivo, puede comprenderse y aplicarse mejor cuando se entiende a la luz de los razonamientos silogísticos. En lo que sigue, se intenta mostrar que algo parecido sucede con las acciones humanas, región propia de la moralidad.

\section{EL SILOGISMO EN RAZONAMIENTO MORAL}

Harry Frankfurt describe los rasgos esenciales de la moralidad cuando dice: "La moralidad tiene que ver, principalmente, con cómo nuestras actitudes y acciones deben de tener en cuenta las necesidades, deseos y derechos de los demás". (Frankfurt, 2004 p.18). En su propósito de enriquecer el marco conceptual de la moralidad, de tal forma que salga del territorio abstracto y apriorístico de las teorías filosóficas, Frankfurt asume la moralidad en relación con los deseos, creencias y actitudes que empujan a las personas a actuar. Este filósofo, siguiendo a Donald Davidson, considera que las razones son los mejores candidatos disponibles para explicar las acciones humanas, pues:

Una razón racionaliza una acción sólo si nos lleva a ver algo que el agente vio, o pensó ver, en su acción; algún rasgo, consecuencia o aspecto de la acción que el agente quiso, deseó, apreció, que le pareció atractivo, benéfico, obligatorio, agradable, o que consideró como su deber.

(Davidson, 1995 p.17)

Así, a diferencia de las causas de los sucesos no humanos, que como ya se describió, se explican a través de leyes y condiciones antecedentes, en el caso de las acciones humanas, son las razones las que explican el por qué las personas han actuado o actuaron como lo hicieron. Compuestas por creencias y deseos, las razones describen lo que empuja a las personas a actuar. Por esto, al asumir Frankfurt las razones como la base expli- cativa de las acciones, para acotar el radio de su filosofía moral, lo que creen las personas y desean es de primera relevancia para comprenderse como agentes morales. Desde una tradición que se remonta a Aristóteles, y que además, toma como base las intuiciones con respecto a los móviles humanos para actuar, Frankfurt se suma a una serie de filósofos contemporáneos, entre los que se destacan Donald Davidson, John Searle, Elizabeth Anscombe, Martha Nussbaum, quienes describen a las razones para actuar como una relación entre creencias y deseos. Así, si Juan toma el vaso que tiene al frente y lo lleva hasta su boca bebiendo el agua que hay en él, esta acción básica se explica apelando a los deseos y creencias de Juan. Él deseaba tomar agua, y tuvo la creencia de que al frente suyo se encontraba un vaso de agua. La creencia y el deseo de Juan se conjugan produciendo la razón que lo lleva a ejecutar la acción de tomar agua.

En tanto la moralidad tiene que ver con las razones y las acciones que llevan a las personas a actuar, comprender la razones para actuar, es comprender en buena parte la estructura básica de la moralidad. Es en este punto en el que la estructura silogística vuelve a ser útil, pues la relación entre creencias y deseos, para formar una razón para actuar, se da esencialmente en el esquema del silogismo. Las creencias y los deseos fungen como las premisas de un razonamiento silogístico, y la conclusión, como la acción, que resulta de la fuerza causal de creencias y deseos. Así, si Juan desea tener una noche tranquila, y Juan sabe que yendo al cine tendrá una noche tranquila, entonces, la acción de Juan ir al cine, puede esquematizarse:

\begin{tabular}{|c|c|}
\hline $\begin{array}{l}\text { El sujeto } X \text { tiene un deseo } \\
\text { El sujeto } X \text { tiene una creencia, relevante } \\
\text { con su deseo }\end{array}$ & Premisas \\
\hline$\%$ El sujeto $X$ actúa & Conclusión \\
\hline
\end{tabular}

La primera premisa del modelo de explicación de las acciones humanas describe el componente motivacional de la conducta humana. Los deseos, ya sean los que causan placer o los que simplemente empujan a cumplir un deber, funcio- 
nan como los fines que se buscan satisfacer. La segunda premisa describe el conocimiento de los medios que son pertinentes para alcanzar el fin. John Searle en su libro Razones para actuar denomina al componente motivacional: motivadores, y al componente cognitivo: efectores. De ahí que, dos tipos de enunciados se funden permitiendo derivar la acción: Los enunciados que describen los motivadores, y los enunciados que describen los efectores. (Searle, 2000 p. 168)

La analogía entre las razones que explican las acciones de las personas y el razonamiento silogístico se trasluce. Dada una información previa, descrita a través de dos premisas, se sigue una conclusión. La conclusión es una consecuencia de las premisas, en tanto las premisas subsumen a la conclusión. Tal como se señalo en el modelo nomológico deductivo, los enunciados que fungen como premisas dentro de las razones para actuar, son diferentes en tanto tienen propiedades distintas. Mientras que los deseos no se evalúan como verdaderos o falsos, tal como se señalo con las leyes, las creencias sí se evalúan como verdaderas o falsas, lo mismo ocurre con las condiciones antecedentes. Esto se debe a que la noción de verdad se aplica a la adecuación entre las proposiciones y los hechos del mundo. Si la creencia representa adecuadamente el estado de cosas del mundo, es verdadera, en caso contrario falsa. Así, si Juan cree que lo que tiene al frente es un vaso de agua, y efectivamente, eso es un vaso de agua, entonces la creencia de Juan será verdadera. Por eso, su deseo de beber agua puede ser satisfecho, porque su conocimiento de los efectores, los medios para colmar su deseo, es verdadero. En lo que respecta a los deseos, estos no son ni verdaderos ni falsos, se satisfacen o no se satisfacen. $\mathrm{Si}$ un deseo no puede cumplirse no significa que el deseo es falso, o si se puede cumplir no significa que sea verdadero.

Cuando se cree se espera que la creencia encaje o se acople al mundo, cuando se desea es lo contrario, se espera que el mundo encaje con el deseo. Estos rasgos de las creencias y los deseos los llama Searle dirección de ajuste. Señala que la dirección de ajuste de las creencias es mente a mundo; es responsabilidad de la creencia encajar con el mundo, en los deseos es mundo a mente; es responsabilidad del mundo encajar con la creencia. (Searle, 2004 p. 94).
Cuando deseamos esperamos que los estados de cosas se acoplen al estado mental. Si deseamos que el domingo por la mañana llueva, es el mundo el que debe encajar con el deseo para que éste sea satisfecho. En el caso de las creencias, son estas las que tiene que encajar con el mundo, el cual es independiente de las mismas. Si tengo la creencia de que el próximo campeón del Mundial de fútbol será Brasil, y mi creencia es correcta, esto es a que ella encajo con el estado de cosas del mundo. (Posada, 2007 p. 65)

En consecuencia, los deseos son satisfechos o insatisfechos, las creencias son verdaderas o falsas. Lo que permite justificar una distinción entre las premisas de los razonamientos que llevan a la acción: cabe la posibilidad de la verdad en un de los componentes dados para derivar o premisas, pero en las otras, dicho valor es inaplicable. Así, dos tipos de enunciados conforman la información dada en los razonamientos para la acción. De estos dos tipos de enunciados se sigue una conclusión, que al afirmarse en los enunciados que fungen como premisas, justifican su derivación. El razonamiento silogístico aparece en las razones para la acción como su esquema estructural. De ahí que, en tanto la moralidad tiene que ver con las maneras como se entienden y evalúan las acciones humanas, comprender las razones para actuar, a partir de su estructura silogística, es promisorio para entender el conocimiento moral.

\section{CONCLUSIONES}

A partir del filósofo René Descartes se señaló que la razón es una característica inherente a la condición humana, a pesar de las críticas recientes a la racionalidad y la verdad. Entendida como la capacidad de derivar de información dada otra información, se recreo desde el razonamiento silogístico descrito por Aristóteles, el proceso prototípico de la racionalidad. Se mostró que en tanto el silogismo describe la relación de inferir de dos premisas una conclusión, su comprensión es promisoria en el interés de establecer los modos de operación de la racionalidad humana. Así, al señalarse dos de los productos intelectuales humanos más depurados, como son la ciencia y la moralidad, se encontró que puede entenderse parte de su estructura básica racional desde el esquema del silogismo. Asimismo, desde la ape- 
lación al modelo nomológico deductivo de Hempel se recreó el rasgo esencial del conocimiento científico: su capacidad explicativa, mostrando que este modelo sigue la estructura del razonamiento silogístico. En tanto la ciencia es explicativa y en el silogismo se da la estructura básica de la explicación, la comprensión de razonamiento silogístico es fecunda en el interés de comprender la actividad científica. De otro lado, se pudo encontrar la misma estructura del silogismo: dos premisas y una conclusión, en la explicación de las razones que llevan a las personas a actuar. Desde Harry Frankfurt se anotó que la moralidad es el compromiso de que las acciones de los individuos tengan en cuenta los intereses y deseos de los demás, y en tanto, las acciones son causadas por razones, el conocimiento de las razones es ineludible en los estudios sobre la moralidad. Las razones se estructuran por creencias y deseos, cada una de estas representa las premisas de un silogismo, que tiene como conclusión la acción. De nuevo se mostró que la estructura formal del silogismo es fértil para comprender un tipo esencial de racionalidad humana: la moral.

En pocas palabras, este trabajo intentó ofrecer desde la estructura formal del silogismo: dos premisas y una conclusión, una imagen común a la ciencia y a la moralidad, que se espera sea promisoria en la necesidad de tener imágenes holísticas sobre las maneras como el hombre ha interpretado racionalmente la realidad.

\section{REFERENCIAS BIBLIOGRÁFICAS}

Aristóteles. Analíticos Primeros. Barcelona: Gredos.

Copi, I. (1962). Introducción a la lógica. Buenos Aires: Eudeba.

Davidson, D. (1995). Ensayos sobre acciones y sucesos. Barcelona: Crítica.

Descartes, R. (1983). Discurso del Método. Barcelona: Orbis. Diez, J. (2002). Iniciación a la lógica. Barcelona: Ariel.

Feyerabend, P. (1985). ¿Por qué no Platón? Madrid: Tecnos

Frankfurt, H. (2004). Las razones del amor, el sentido de nuestras vidas. Barcelona: Paidós.

(2007). Sobre la verdad. Barcelona: Paidós.

Hempel, C. (1996). La explicación científica. Barcelona: Paidós.

Nagel, E. (1991). La estructura de la ciencia. Barcelona: Paidós.
Perelman, Ch. (2000). Tratado de la Argumentación, la nueva retórica. Madrid: Gredos.

Popper, K. (1996). La lógica de la investigación científica. México: Rei.

Posada, G. (2007). La Noción tripartite del conocimiento, una introducción a la epistemología. Manizales: Universidad de Caldas.

Rorty, R. (2000). El pragmatismo una versión. Barcelona: Ariel. Salmon, W. (1965). Lógica. México: Editorial Hispano Americana.

Searle, J. (2004). Mente, lenguaje y sociedad. Madrid: Alianza.

Searle, J. (2000). Razones para actuar. Madrid: Círculo de lectores.

Thomson, G. (2000). On Aristotle. Belmont: Wadsworth.

Von Wright, G. H. (1980). Explicación y comprensión. Madrid: Alianza.

Wittgenstein, L. (1988). Investigaciones filosóficas. México Crítica. 